\title{
Modeling the Effect of Temperature on the Nanotube Field Effect Transistors Using Neural Network
}

\author{
Menacer Farid $^{1}$ and Kadri Abdelmalek ${ }^{2}$ \\ Electronics Department, University of Batna, Algeria \\ ${ }^{1}$ menacer_farid@yahoo.fr
}

\begin{abstract}
In this article, we modelled and simulated Carbon Nanotube Field Effect Transistors (CNTFET); this transistor has a carbon nanotube channel. An Artificial Neural Networks, (ANN) model was developed to reproduce accurately the behavior of CNTFET to predict its response for a wide range of temperature and voltage with focusing on the experimental data. The neural model is tested and validated; hence the ANN modet is able to predict the CNTFET behavior with good accuracy. Finally, the proposed ANN model is integrated as a component in the library of simulation softmare of PSPICE electronic components.
\end{abstract}

Keywords: Carbon Nanotube Field Effect Transistors (CNTFEY), modeling, thermal effect, Artificial Neural Networks (ANN), PSPICE, Analog Behavioral Modeling (ABM)

\section{Introduction}

The MOSFETs (Metal Oxide Semiconductor Field Effect Transistor) components had large applications over the last decade. Due to the effects of short channel during the process of miniaturization; scientists werê forced to seek different models for the fieldeffect transistor, so that these shall become free from these drawbacks. Among these models, we may mention the Carbon Nanotube (CNT) model. The field-effect transistor, having the active reg on as carbon nanotube has better performance than the ordinary MOSFET [1].

The short channel effects in the MOSFET, appearing during miniaturization, are reduced or very weak in the Carbon Nanotube of Field Effect transistor (CNTFETs). Thus, we cango further with the miniaturization of the device, so that the density of the integrated circuit can be increased [2].

A CNTFET is an FET that has, instead of silicon as a channel material in the traditional structure of the MOSFET, a single or set of CNTs. The CNT has a significant importance in applications ofelectronic components; its faculties make it as a promising material [3]. Its nano structural size has many interesting and often unexpected faculties, therefore there are plenty of opportunities for their use in nanotechnology.

We have considered in this paper, particularly for the Conventional CNTFET denoted as CCNTFET, with highly doped source and drain contacts; these devices show better performances in terms of current report "on-off" and sub threshold slope [4-5]. Figure 1 shows a 2D representation of C-CNTFET which the conduction behavior is similar to an ordinary MOSFET one [6]. 


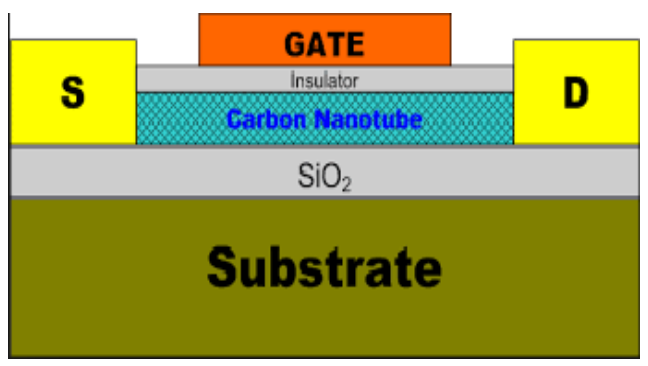

Figure 1. 2D Representation of C- CNTFET

The carbon nanotube requires a large investment before it can reach the desirable quality for the production of components. Prior to such an investment, it is necessary to address an extensive_study on electronic properties of the material. In order to consider the effect of temperature on the I-V characteristics of CNTFET, we propose a thermal model of CNTFET using neural network approach.

The main objective of this study is to model a C-CNTFET by means of neurat network; this model predicts the I-V characteristics of CNTFET for different temperatures, based on results got by [3]; the ANN model is implemented as a component in a PSPICE electric simulator library, this component reproduces faithfully the C-CNTFET behavior.

\section{Artificial Neural Networks (ANN)}

The artificial neural network is a means- of calculation that attempts to simulate the structure and functionality of the biological neuron networks [7-8].It is a powerful data modelling tool, which can capture and represent complex relationships of input / output through some internal calculations. The most common model of the neural network is the Multilayer Perceptron (MLP) [9], 88 show in Figure2.

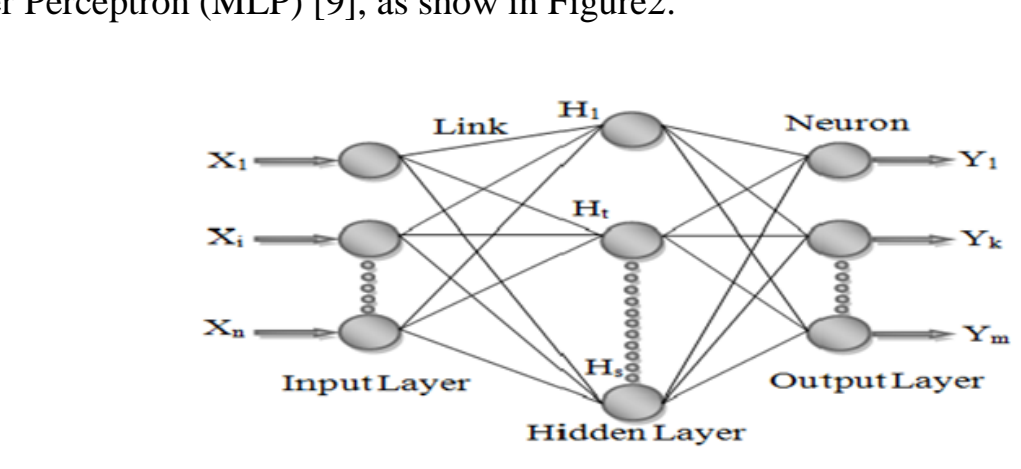

Figure 2. MLP Structure

Each link has a weight parameter corresponding thereto; each neuron is composed of a set of mputs and an activation function.

The-architecture of the network consists of an input layer (X), an output layer (Y) and one or more hidden layers (H). MLP is a model that maps a set of input data on an appropriate set of outputs; a simplified overview of the proposed ANN model is shown in Figure 3. 


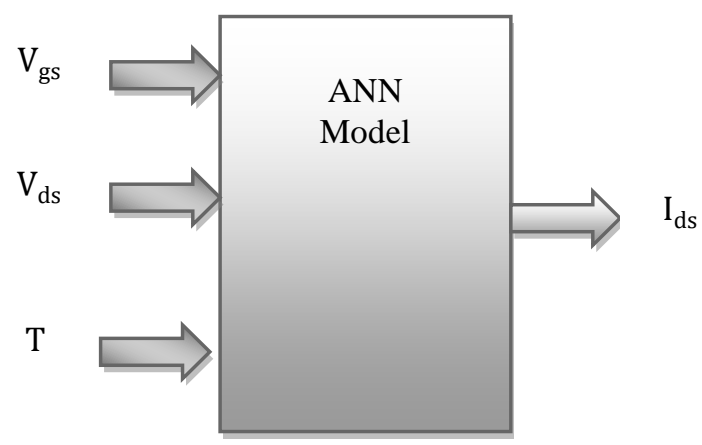

Figure 3. Simplified Overview of ANN Model

Equation 1 shows the algorithm generally used to compute the output of an artificial neuron. The output of each neuron $f$ depends on the activation value that is the weighted sum of the inputs [10], where $w_{i}$ is the weight-related input $x_{i}$ and $f>$ is the activation function.

$$
f(x)=\left(\sum\left(x_{i} * w_{i}\right)\right)
$$

The MLP are trained with the standard back-propagation algorithm, which compares the output provided with the aimed output, wherein the mean square error is calculated. If the mean square error is more than a prescribed threshold value, it Is backward propagated of the output to the input, and the weight will still be changed until the error, or number of iterations is in a prescribed limit.

Training a network is a process where all of adjusted parameters, weights and biases, are optimized to make the best prediction of the target ariable on the basis of background variables.

To compare the results of the proposed ANN model with experimental results, we use the measure of relative erron(RE) expressêd as a percentage and defined by the following relationship [11], where $\mathrm{I}_{\text {pred }}$ is the predicted drain current based on ANN, $\mathrm{I}_{\exp }$ shows the experimental current.

$$
\mathrm{RE} \%=\frac{\operatorname{Lex}_{\mathrm{R}}-\mathrm{I}_{\text {pred }}}{I_{\exp }} \times 100
$$

The Middle Relative Error (MRE) is given by [12], where $\mathrm{N}$ is the number of data (database size)

$$
\mathrm{MRE}=\frac{1}{\mathrm{~N}} \boldsymbol{\Sigma}_{1}=1 \mathrm{RE} \mathrm{I}_{\mathrm{i}}
$$

MLP networks are trained with the standard back-propagation algorithm by attempting to minimize the error.

\section{C-CNTFET Modeling using Neural Networks}

The output of the network is the drain current $I_{d s}$, the training data consists of the set of representative points got from the experimental measurements [13] is show in Figure 4. 


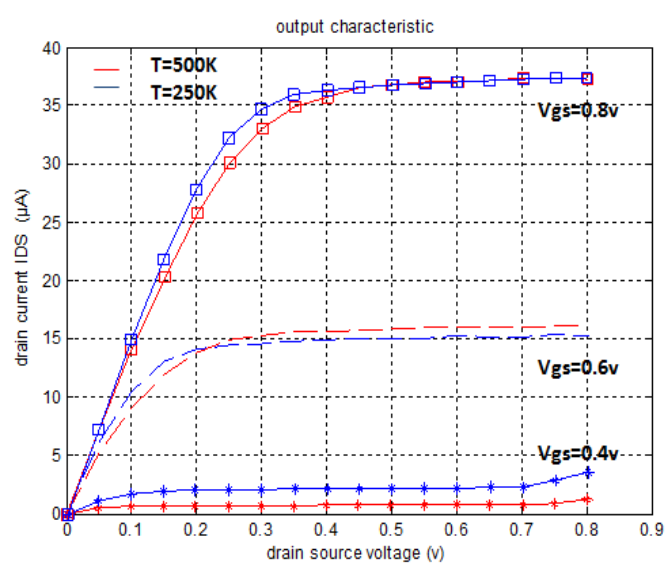

Figure 4. Experimental Output characteristic $I_{d s}$ According to $V_{d s}$ for Different $V_{g s}$ and Temperature $T$ values [13]

After choosing the type of network, we shall have to find an optimal architecture of neural network (the number of hidden layers, the number of neurons in each/ayer and the activation function for each layer).

Learning and optimization of previous networ is accomplished through a structured program in MATLAB, Figure 5 shows the flowchan of the steps followed

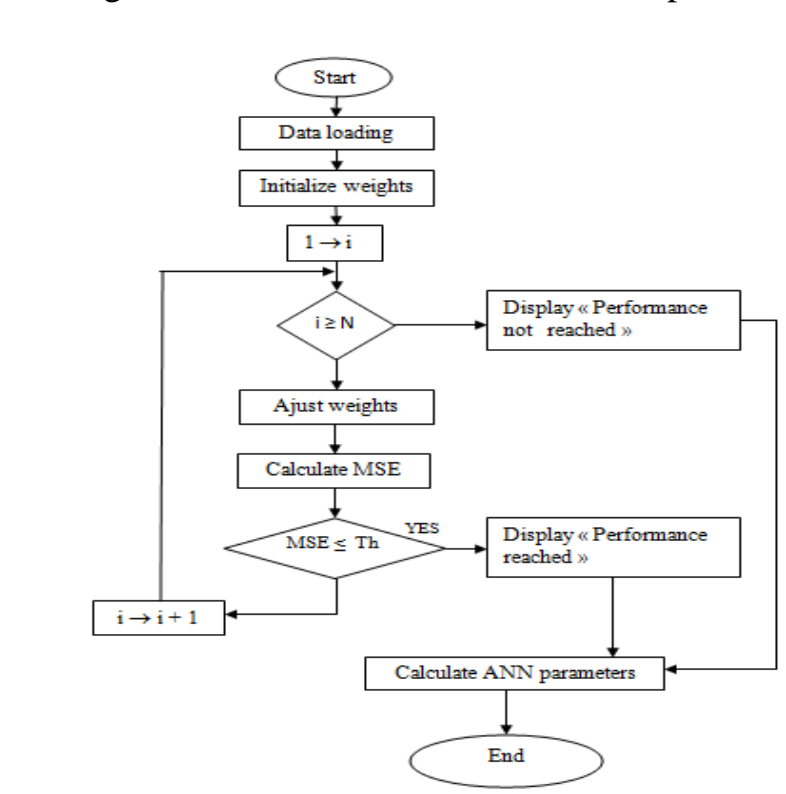

Figure 5. Flowchart Training and Optimization

The optimized network parameters for modeling the C-CNTFET are summarized in Table 3. 


\section{Table 3. Optimized Parameters of the Neural Networks Model}

\begin{tabular}{|c|c|c|c|c|}
\hline \multirow{3}{*}{ Database } & \multicolumn{2}{|c|}{ Training base } & \multicolumn{2}{|c|}{380} \\
\hline & \multicolumn{2}{|c|}{ Test base } & \multicolumn{2}{|c|}{190} \\
\hline & \multicolumn{2}{|c|}{ Validation base } & \multicolumn{2}{|c|}{190} \\
\hline \multirow{4}{*}{ Number of Neurons } & \multicolumn{2}{|c|}{ Input layer } & \multicolumn{2}{|c|}{3} \\
\hline & \multicolumn{2}{|c|}{$1^{\text {st }}$ hidden layer } & \multicolumn{2}{|c|}{5} \\
\hline & \multicolumn{2}{|c|}{$2^{\text {nd }}$ hidden layer } & \multicolumn{2}{|c|}{4} \\
\hline & \multicolumn{2}{|c|}{ Output layer } & \multicolumn{2}{|c|}{1} \\
\hline \multirow{3}{*}{ Transfer function } & \multicolumn{2}{|c|}{$1^{\text {st }}$ hidden layer } & \multicolumn{2}{|c|}{ Logsig } \\
\hline & \multicolumn{2}{|c|}{$2^{\text {nd }}$ hidden layer } & \multicolumn{2}{|c|}{ Logsig } \\
\hline & \multicolumn{2}{|c|}{ Output layer } & \multicolumn{2}{|c|}{ linear } \\
\hline \multirow[t]{3}{*}{ Input } & \multicolumn{2}{|c|}{$\mathrm{T}\left({ }^{\circ} \mathrm{C}\right)$} & $\mathrm{V}_{\mathrm{ds}}(\mathrm{V}$ & $\mathrm{V}_{\mathrm{gs}}(\mathrm{v})$ \\
\hline & $\begin{array}{c}\mathrm{M} \\
\mathrm{ax}\end{array}$ & $500 \mathrm{k}$ & 0.9 & 0.8 \\
\hline & $\begin{array}{c}\mathrm{M} \\
\text { in }\end{array}$ & $250 \mathrm{k}$ & 0 & 0.4 \\
\hline \multirow{3}{*}{ Output } & \multicolumn{4}{|c|}{$\mathrm{I}_{\mathrm{ds}}(\mu A)$} \\
\hline & \multicolumn{2}{|c|}{ Max } & 38 & \\
\hline & \multicolumn{2}{|c|}{ Min } & & \\
\hline \multicolumn{5}{|l|}{ Test MSE } \\
\hline Training MSE & & & & \\
\hline
\end{tabular}

According to Figure 6, there is a good accuracy of the correlation factor $(\mathrm{CF}=0.997)$ between the ANN outputs ( $\mathrm{I}_{\text {pred }}$ : predicted) and corresponding targets ( $\mathrm{I}_{\mathrm{exp}}$ : experimental).

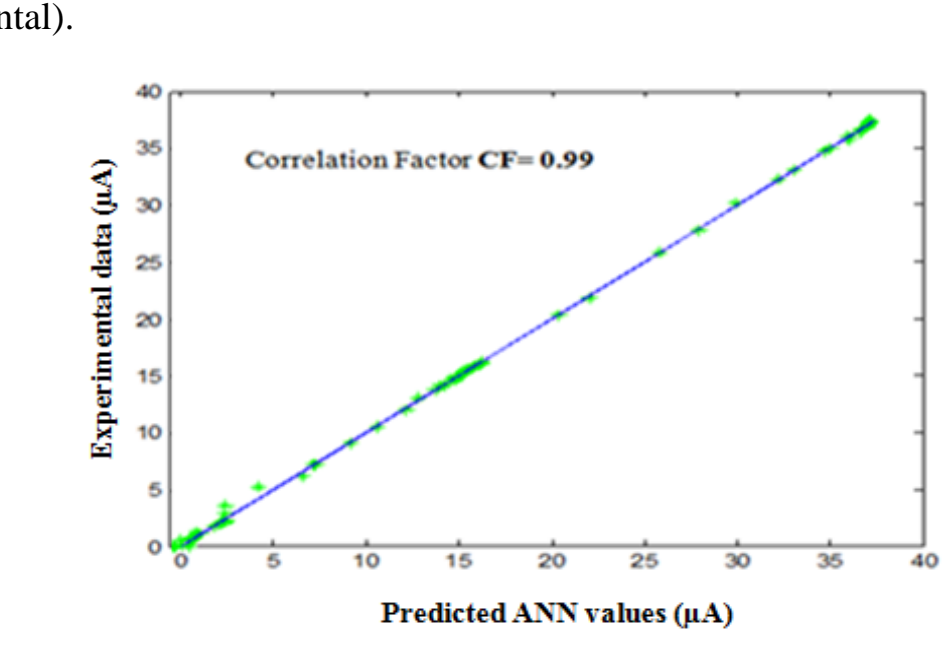

Figure 6. Comparison of the Experimental Data and Predicted (ANN) Results for Training Data

Figure 7 shows the output current $\mathrm{I}_{\mathrm{ds}}$ of CNTFET depending on the voltages $\mathrm{V}_{\mathrm{gs}}$ and $V_{\mathrm{ds}}$ at different temperatures $(250 \mathrm{~K}$ and $500 \mathrm{~K})$. The gate and the drain voltages are chosen so that we may make a comparison between the experimental results published in [13] and our simulations. 


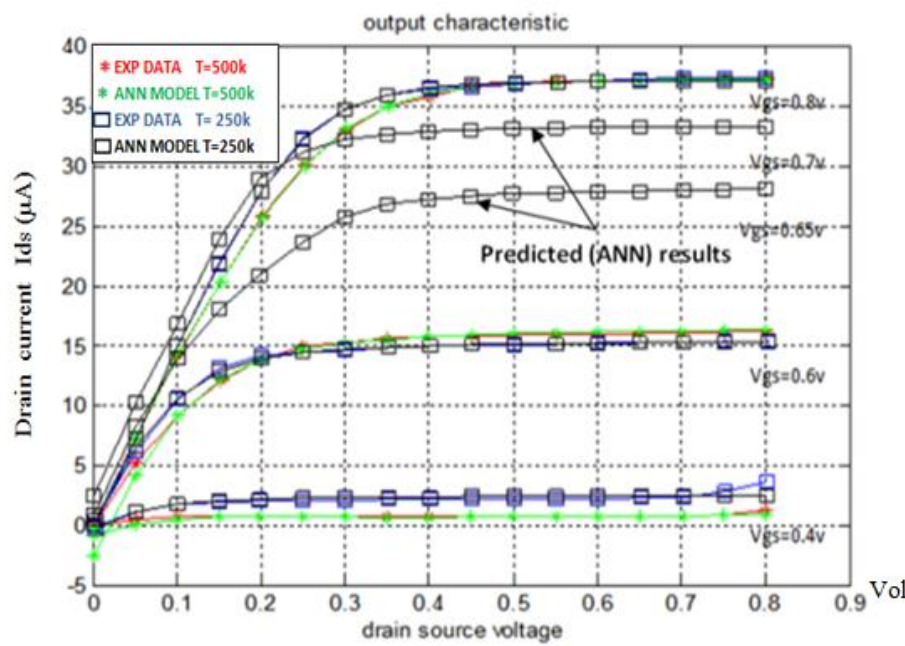

Figure 7. Output Characteristics of ANN Model and Experimental Data

The Simulations show consistency and relatively good compatibility, particularly for high gate voltages $\left(\mathrm{V}_{\mathrm{gs}}\right)$. In addition to that our neuronal model has the potential to predict the behavior of CNTFET when it is subjected to newconditions, with no need to resort to experimental; the values of $\mathrm{V}_{\mathrm{gs}}$ must be in the interval $\left[\mathrm{V}_{\mathrm{gs}}{ }_{\text {min }}, \mathrm{V}_{\mathrm{gs}}\right.$ max which is illustrated in the Table $1\left(\mathrm{~V}_{\mathrm{gs}}=0.65\right.$ volts, $\mathrm{gs}=0.7$ volts $)$.

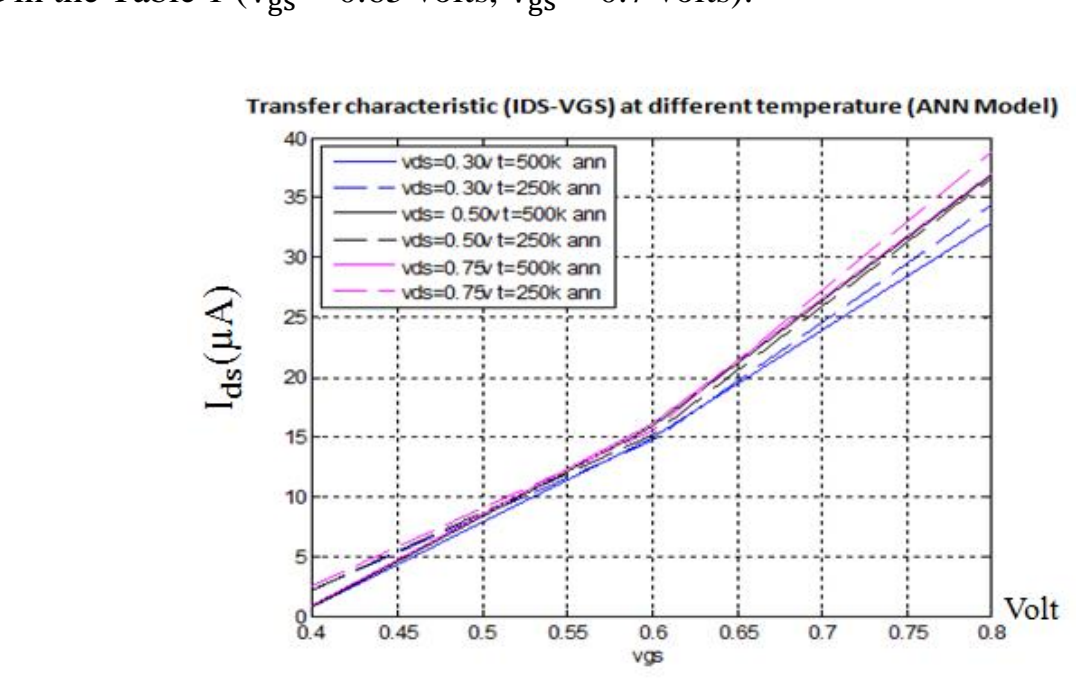

\section{Figure 8. Transfer Characteristic of ANN Model for Different Temperature and Different $V_{d s}$}

Figure 8 shows the transfer characteristics of the C-CNTFET ANN model for various values of voltage $V_{d s}$ and temperature. We observed that the transfer characteristic of ANN model for different temperature and different $V_{\mathrm{ds}}$ has a nearly linear shape.

\subsection{Implementation of the Model ANN on PSPICE}

To validate our proposed neural model, we implemented it in the library of the PSPICE simulator. The results got from of the optimal architecture of the ANN, like bias and weight, are used for the implementation of a model that will be used as a component in the library of PSPICE. 
The use of ABM (Analog Behavioral Modeling) boxes from the PSPICE library helps to implant the ANN model in this simulator. Figure 9 shows the ABM architecture, our model has 13 ABM boxes, each neuron ANN is replaced by an ABM box that characterizes this neuron.

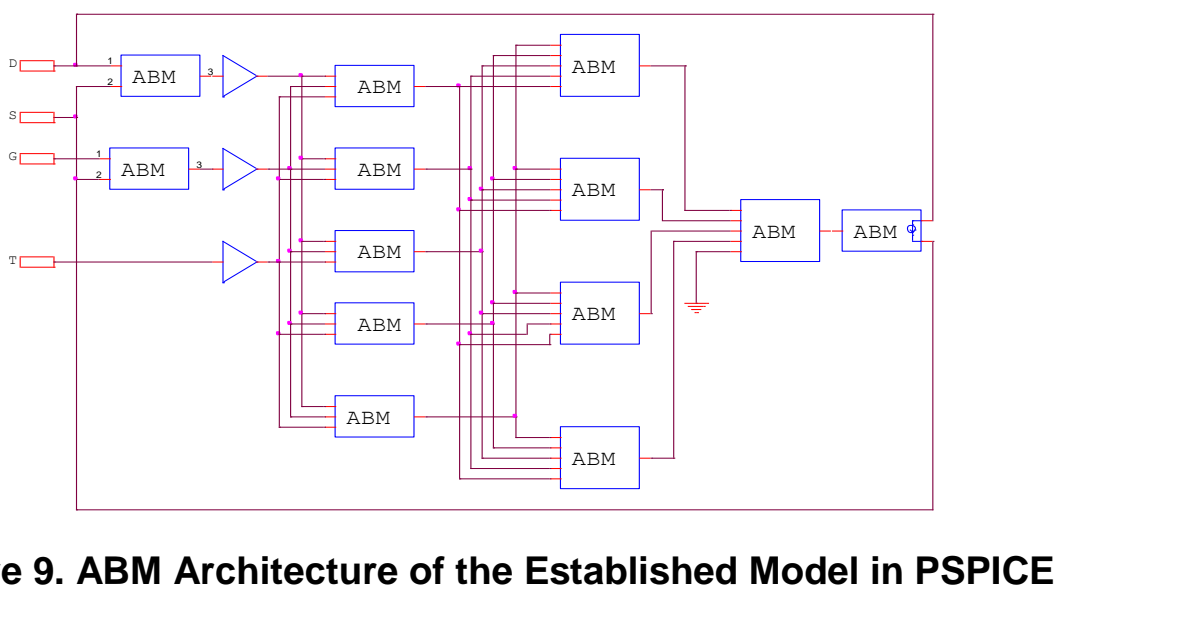

In Order to got the $I_{d s}$ output characteristics according to $V_{d s}$ (outpul characteristic) for different values of $\mathrm{V}_{\mathrm{gs}}$ voltage and temperature, the PSPICE software allowed us to draw the following curves; we just need to apply the appropriate alues of $\mathrm{V}_{\mathrm{ds}}, \mathrm{V}_{\mathrm{gs}}$ and $\mathrm{T}$ to our circuit inputs Figure 10.

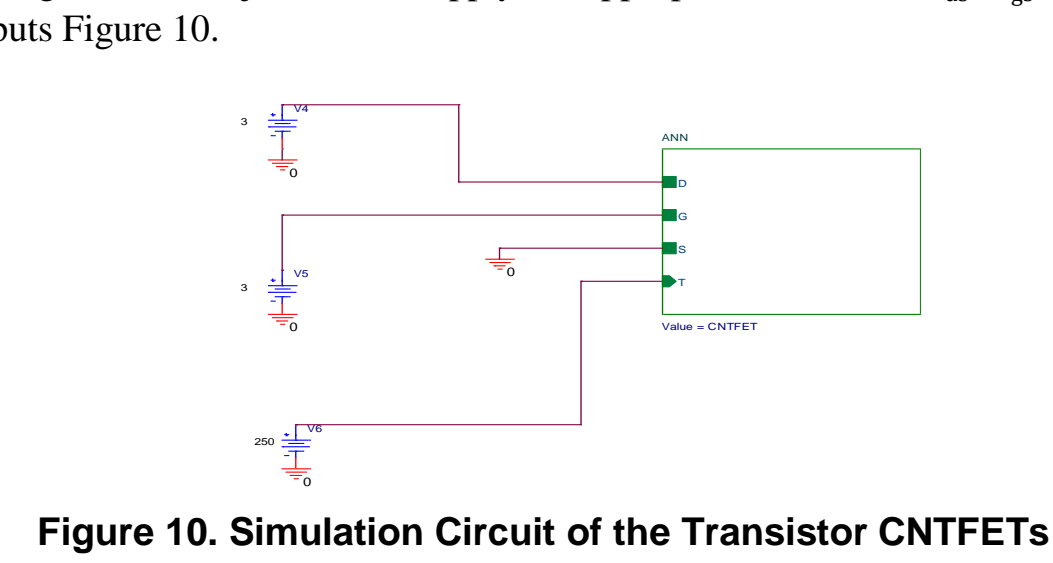

By a DC-SWEEP analysis, for temperatures $\mathrm{T}=250 \mathrm{~K}$ and $\mathrm{T}=500 \mathrm{~K}$, while varying the $\mathrm{V}_{\mathrm{ds}}$ voltage of 0 volt to 1 volt with a step of 0.1 volt, and for $\mathrm{V}_{\mathrm{gs}}$ which takes the values 0.4 volt, 0.6 volt and 0.8 volt, we get the curves plotted in Figure 11. It is observed that there is a good harmony between the result got through the experimental and that got through the PSPICE simulator. 


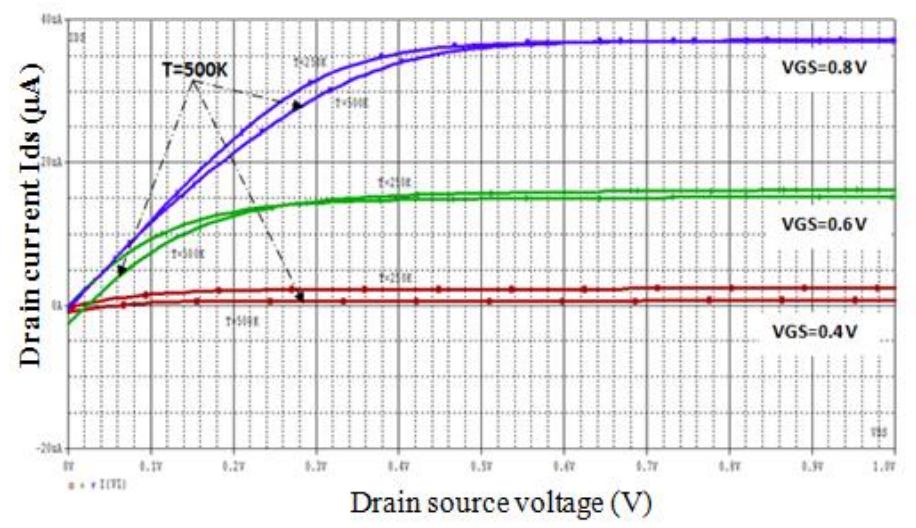

Figure 11. Current-Voltage Characteristics $\left(I_{d s}, V_{d s}\right)$ from the ANN-Pspice Model $(\mathrm{T}=500 \mathrm{~K}, \mathrm{~T}=250 \mathrm{~K})$

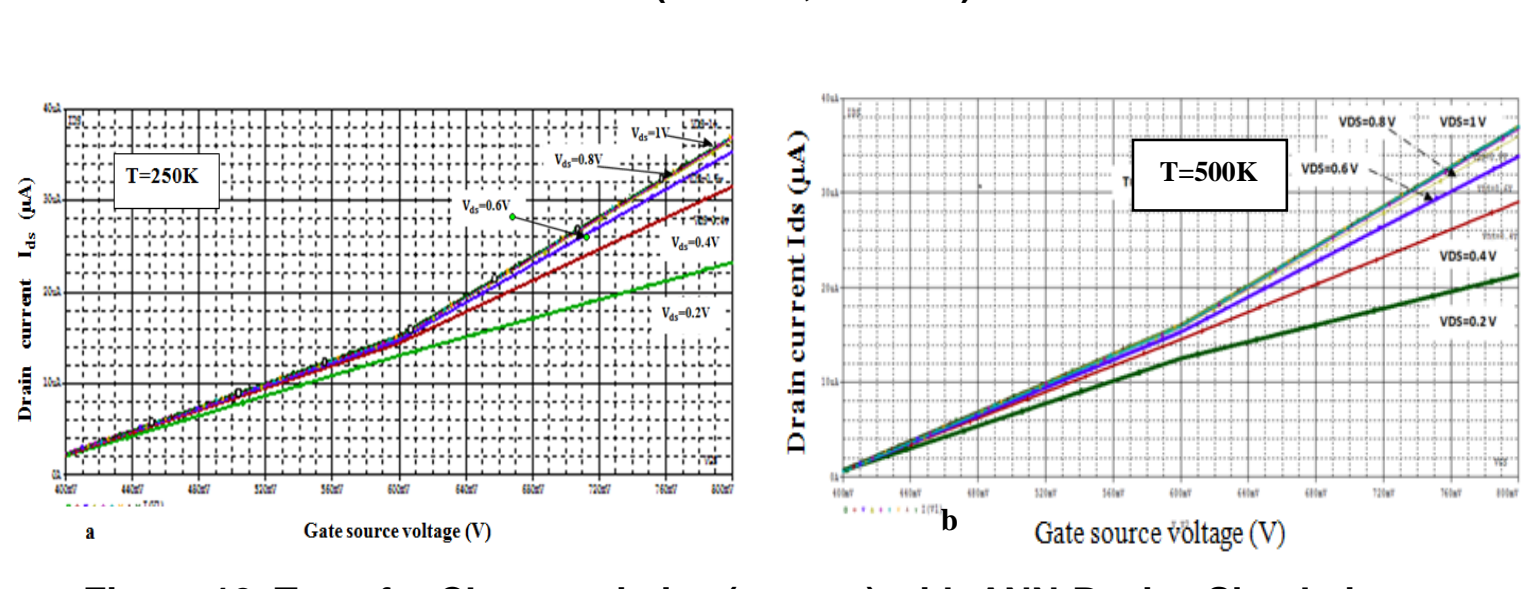

Figure 12. Transfer Characteristîcs $\left(I_{d s}-V_{g s}\right)$ with ANN-Pspice Simulations

By analogy with first test made to get the characteristic of the transfer, and by a DCSWEEP analysis for temperatures $T=250 \mathrm{~K}$ and $\mathrm{T}=500 \mathrm{~K}$, while varying the $\mathrm{V}_{\mathrm{gs}}$ voltage from 0.4 volt to 6.8 volt, and for $V_{\mathrm{ds}}$ ranging from 0.2 volts to 0.8 volts, with a step of 0.2 volt, we derive the curves plotted in Figures 12-a and 12-b.

In order to demonst ate the effectiveness of our neural model, we propose the study and simulation of a oscillator and a nano-inverter circuit; the latter is regarded as the most important block in the design of VLSI (Very Large Scale Integration) digital circuits.

\subsection{PSPICE Input / Output Signals of the ANN Model of Inverter}

In order to obtain the PSPICE input / output signals of our neural inverter, we use the scheme presented in Figure 13. 


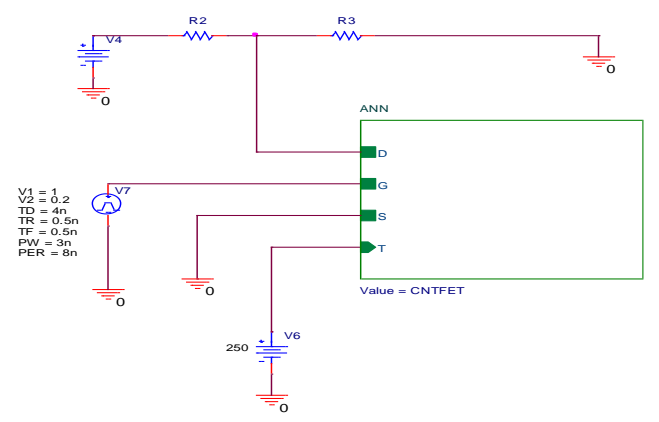

\section{Figure 13. Neural Model of the Inverter Circuit}

The input and output signals of our inverter based on the proposed neural model are represented in the Figures14 where $\mathrm{V}_{\mathrm{ds}}=5$ Volt and $\mathrm{T}=250 \mathrm{~K}$, it can be remarked that the operation of the inversion has been well performed.

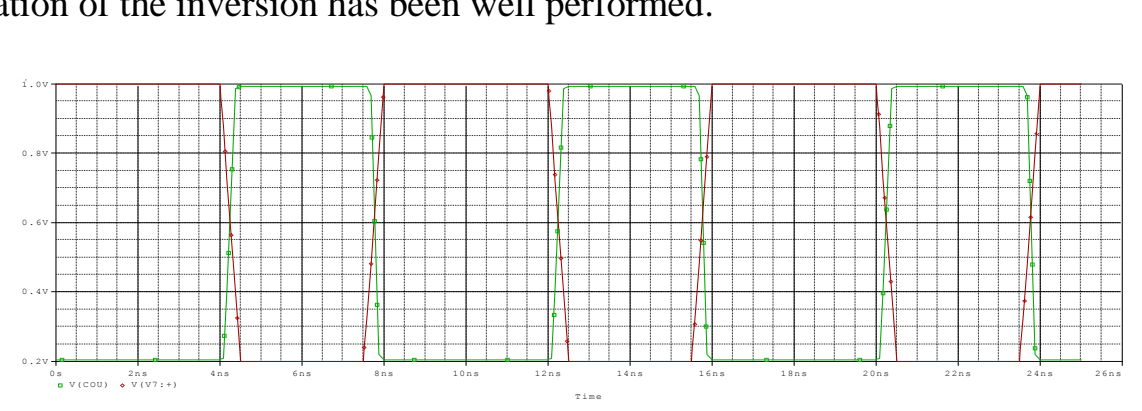

Figure 14. Response of Inventer $\mathrm{T}=250 \mathrm{~K}$

\subsection{PSPICE Input and Output Signals of the Ring Oscillator ANN Model}

The ring oscillator is made up of several inverters in series. The inverter is formed by a neural block, as shown in Figure 13. The output of the last inverter feeds the input of the first inverter; that allows the circuit to oscillate, as shown with PSPICE simulation in Figure 15.

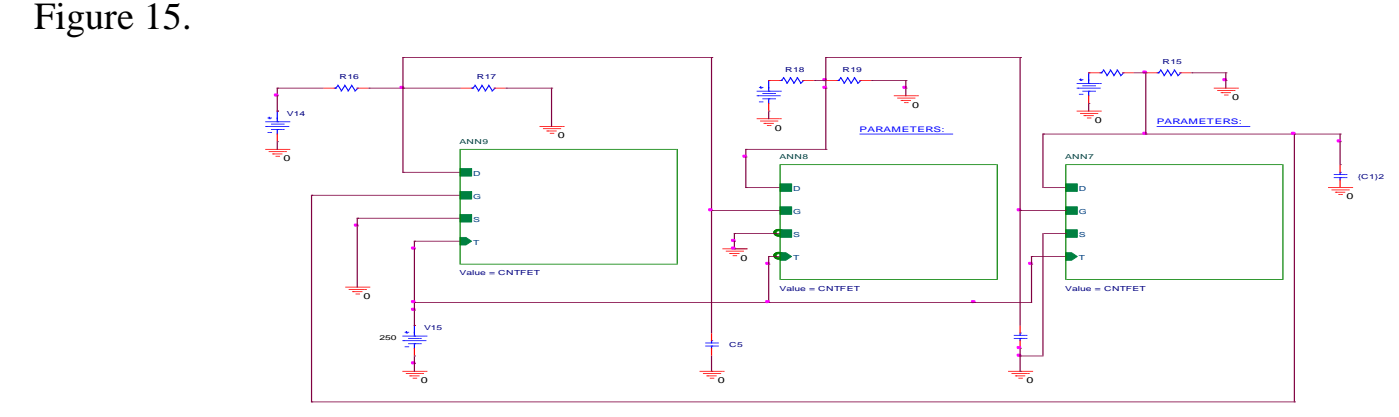

Figure 15.The Ring Oscillator by CNTFET ANN Model 


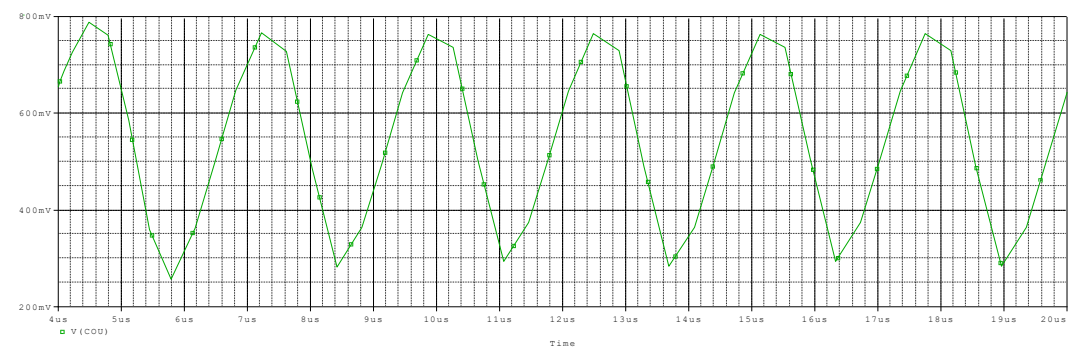

\section{Figure 16.The Response of the Ring Oscillator Based ANN CNTFET Model}

Figure 16 show the output signal of the ring oscillator based on the neural model, we observed a well-functioning of the proposed ring oscillator.

\section{Conclusion}

In this paper we modeled the CNTFET using artificial neural networks, the neural model is tested and validated, and this model reproduces faithfully the behavior of CNTFETs taking into consideration the effect of temperature,

In addition, the ANN model is able to predict the behavior of CNTEET in previously unknown circumstances.

The results (optimal architecture, bias, and weights of the network) were then exploited in the implementation of the model as a component in the PSPICE simulator library. The component has reproduce faith ully the CNTFET behavior

The simulations carried out in PSPICE based, on the ANN model were presented as inverter and oscillator rings.

\section{References}

[1] R. Marani and A. G. Perni, Modelling and implementation of subthreshold currents in schottky barrier CNTFETs for digital applications", International Journal of Research and Reviews in Applied Sciences, vol. 11, issue 3, (2012) pp. 368-376.

[2] Md. A. Kabir, T. Nandy, M. A Haque, A. Dutta and Z. H. Mahmood, "Performance Analysis of Cntfet and Mosfet Focusing Channel Length, Carrier Mobility and Ballistic Conduction in High Speed Switching", International Journal of Advances in Materials Science and Engineering (IJAMSE), vol. 3, no. 3/4, (2014), pp. 1-11.

[3] R. Maranı and A. G. Perrı, "DC thermal modeling of CNTFETs based on a semi-empirical approach", International Journa of Advances in Engineering and Technology (IJAET), vol. 8, issue 2, (2015), pp. $38-45$.

[4] A. Javey, H. Kin, M. Brink, Q. Wang, A. Ural, J. Guo, P. McIntyre, P. McEuen, M. Lundstrom and H. Dai , "High- $\kappa$ dielectrics for advanced carbon-nanotube transistors and logic gates", Nature Materials, vol. 1, no 4, (2002), pp. 241-246.

[5] S. G Shirazi and S. Mirzakuchaki, "High on/off current ratio in ballistic CNTFETs based on tuning the gate insulator parameters for different ambient temperatures”, Applied Physics A, vol. 113, issue 2, (2013), pp. 447-457.

6] -R. Marani and A. G. Perri, “A simple model of Carbon Nanotube Field Effect Transistors”, International Journal of Advances in Engineering \& Technology, vol. 6, issue 3, (2013), pp. 1076-1082.

[7] A. Rantala, S. Franssila, K. Kaski, J. Lampinen, M. Aberg and P. Kuivalainen, "Improved neuron MOStransistor structures for integrated neural network circuits", IEE Proceedings - Circuits, Devices and Systems, vol. 148, issue 1(2001), pp. 25-34.

[8] A. F. Abo-Elhadeed, "Modeling Ballistic Double Gate MOSFETs using Neural Networks Approach", Proceedings of the 8th Spanish Conference on Electron Devices, CDE, (2011), pp. 1-4.

[9]M. Hayati and F.Mozafari, "Modeling and Simulation of Top-Contact Pentacene Thin Film Transistor Using Artificial Neutral Networks", Australian Journal of Basic and Applied Sciences, (2012), pp. 242250.

[10] B. Yegnanarayana, “Artificial Neural Networks”, Prentice-Hall of India Pvt.Ltd, (2004). 
[11] M. Hayati , A. Rezaei and M. Seifi, "CNT-MOSFET modeling based on artificial neural network: Application to simulation of nanoscale circuits", Solid-State Electronics, vol. 54, no. 1, (2010), pp. 5257.

[12] M. Hayati, M. Seifi and A. Rezaei, "Double Gate MOSFET Modeling Based on Adaptive Neuro-Fuzzy Inference System for Nanoscale Circuit Simulation”, ETRI Journal, vol. 32, no. 4, (2010), pp. 530-539

[13] A. Naderi, P. Keshavarzi and H. Elahipanah, "The Impact of Varying Temperature on Performance of Carbon Nanotube Field-Effect Transistors", Proceedings of the 9th WSEAS International Conference on Microelectronics, Nanoelectronics, Optoelectronics, (2010), pp. 56-60.

\section{Authors}

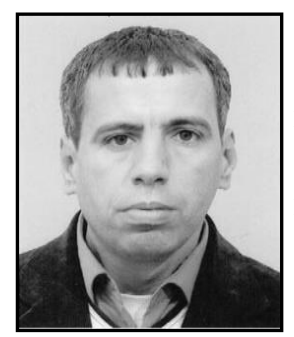

Menacer Farid, he received the engineer degrees in electronics control and master degrees in instrumentation from Batna University in 1996 and 20011, respectively

His research interests include neuronal networks, sensors and intelligent system.

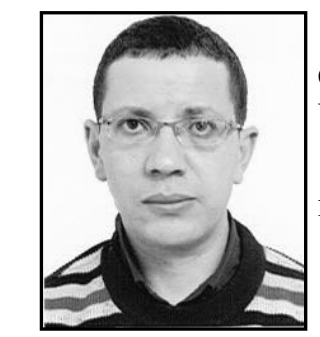

Kadri Abdelmalek, he recenved the engineer degrees in electronics control and master degrees in instrumentation from Batna University in 1995 and 20012, respectively.

His research interests include neuronal networks, sensors, intelligent system and nanotechnology

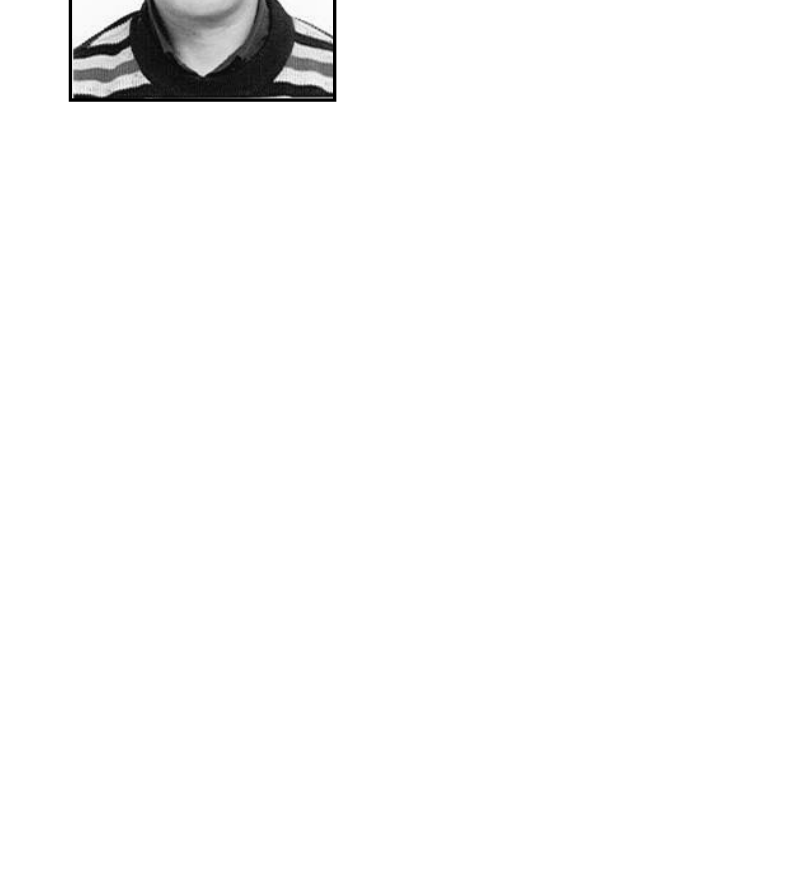


International Journal of Hybrid Information Technology

Vol. 9, No.10 (2016)

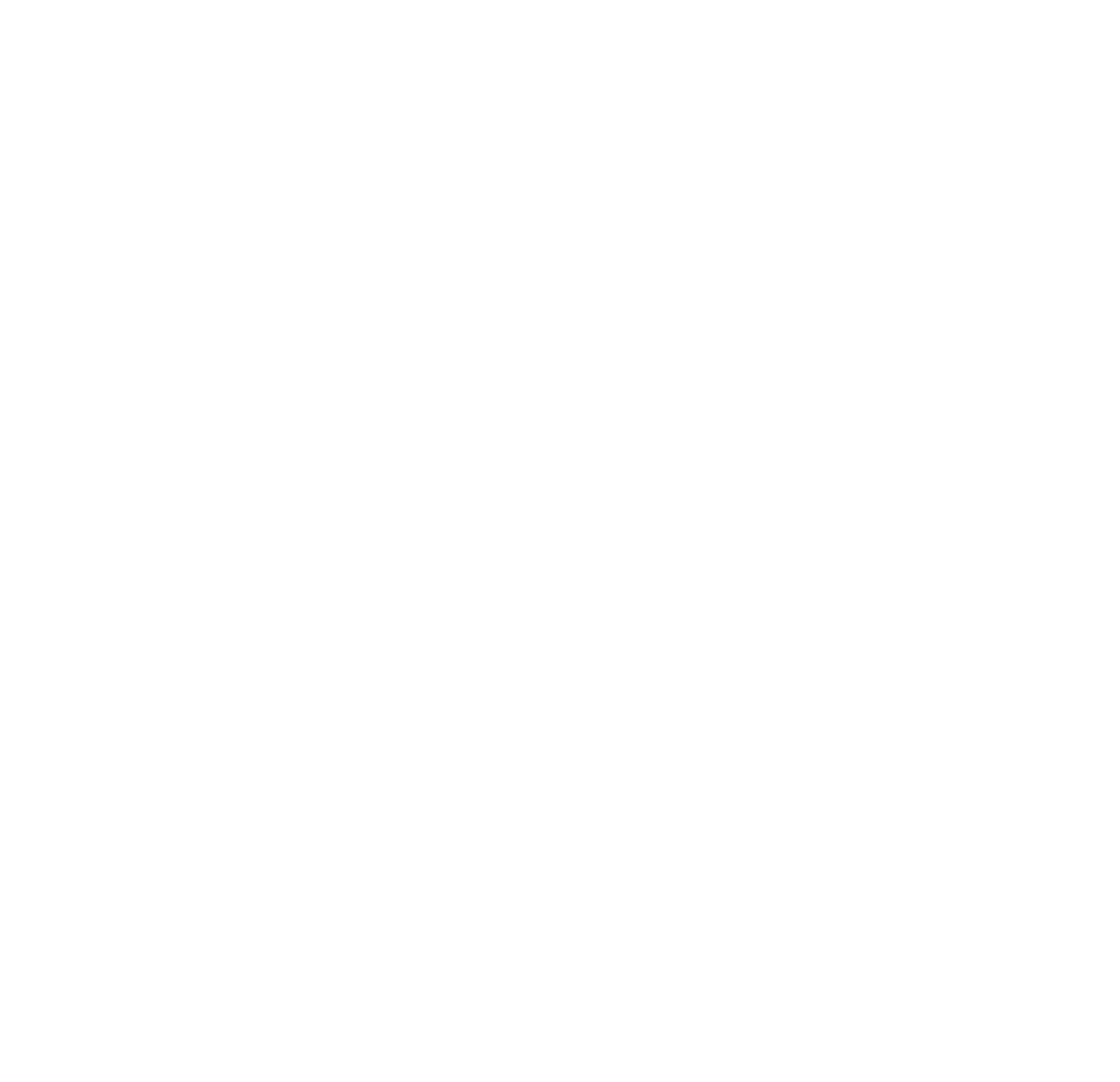

